Cahiers $d u$ MONDE RUSSE

\section{Cahiers du monde russe}

Russie - Empire russe - Union soviétique et États indépendants

$43 / 2-3 \mid 2002$

Contacts intellectuels, réseaux, relations internationales

\title{
Aleksej Jakovlevič Polenov à l'université de Strasbourg (1762-1766)
}

L'identité naissante d'un intellectuel

Wladimir Berelowitch

\section{OpenEdition}

\section{Journals}

Édition électronique

URL : https://journals.openedition.org/monderusse/8495

DOI : 10.4000/monderusse.8495

ISSN : $1777-5388$

Éditeur

Éditions de l'EHESS

Édition imprimée

Date de publication : 1 avril 2002

Pagination : 295-320

ISBN : 2-7132-1781-4

ISSN : $1252-6576$

Référence électronique

Wladimir Berelowitch, « Aleksej Jakovlevič Polenov à l'université de Strasbourg (1762-1766) », Cahiers

du monde russe [En ligne], 43/2-3 | 2002, mis en ligne le 01 janvier 2007, consulté le 04 septembre

2022. URL : http://journals.openedition.org/monderusse/8495 ; DOI : https://doi.org/10.4000/

monderusse. 8495 


\title{
CAIR N
}

chercher : repérer : avancer

Cet article est disponible en ligne à l'adresse :

http://www.cairn.info/article.php?ID REVUE=CMR\&ID NUMPUBLIE=CMR 432\&ID ARTICLE=CMR 4320295

\section{Aleksej Jakovlevic Polenov à l'université de Strasbourg (1762-1766). L'identité naissante d'un intellectuel par WLADIMIR BERELOWITCH}

\author{
| Editions de l'EHESS | Cahiers du monde russe
}

\section{2/2-3 - Vol 43}

ISSN 1252-6576 | ISBN 2713217814 | pages 295 à 320

\footnotetext{
Pour citer cet article :

- BERELOWITCH W., Aleksej Jakovlevic Polenov à l'université de Strasbourg (1762-1766). L'identité naissante d'un intellectuel, Cahiers du monde russe 2002/2-3, Vol 43, p. 295-320.
}

Distribution électronique Cairn pour les Editions de l'EHESS.

(C) Editions de l'EHESS. Tous droits réservés pour tous pays.

La reproduction ou représentation de cet article, notamment par photocopie, n'est autorisée que dans les limites des conditions générales d'utilisation du site ou, le cas échéant, des conditions générales de la licence souscrite par votre établissement. Toute autre reproduction ou représentation, en tout ou partie, sous quelque forme et de quelque manière que ce soit, est interdite sauf accord préalable et écrit de l'éditeur, en dehors des cas prévus par la législation en vigueur en France. Il est précisé que son stockage dans une base de données est également interdit. 


\title{
ALEKSEJ JAKOVLEVIČ POLENOV À L'UNIVERSITÉ DE STRASBOURG (1762-1766)
}

\author{
L'identité naissante d'un intellectuel
}

Y eut-il des « intellectuels » dans la Russie du XVIII siècle et si oui, comment les situer et les définir ? Cette question a fait l'objet de nombre d'études anciennes et nouvelles sur lesquelles il n'y a pas lieu de revenir ici ${ }^{1}$. Rappelons également que, depuis une ou deux décennies, l'histoire des intellectuels en Europe a été considérablement renouvelée et qu'en particulier, certains historiens ont refusé d'appliquer cette notion à des époques antérieures à la fin du XIXe siècle. En schématisant cette littérature déjà abondante, on pourrait dire que nous avons aujourd'hui le choix entre une définition des intellectuels limitée à une catégorie qui a surgi dans la

\footnotetext{
1. Comme on le sait, il faut distinguer, au moins dans un premier temps, la notion d'« intellectuels » et celle d'intelligencija, ce que font généralement les historiens occidentaux, qui voient dans cette dernière une réalité russe spécifique, tandis que les historiens russes (et de façon générale les auteurs russes depuis la seconde moitié du XIXe siècle) ne font aucune différence sensible entre les deux notions. Pour la période qui nous intéresse, contentons-nous ici de citer l'ouvrage essentiel de Marc Raeff, Origins of Russian intelligentsia : Eighteenth-century nobility, New York, 1966, auquel il faut ajouter, pour ses qualités informatives et certaines directions de recherche, celui de Mihail M. Štrange, Demokratičeskaja intelligencija Rossii $v$ XVIII veke (L'intelligentsia démocratique en Russie au XVIII' siècle), Moscou, Nauka, 1965. Michael Confino, dans son article : « Idéologies et mentalités : intelligentsia et intellectuels en Russie aux XVIII-XIXe siècles» (reproduit dans id., Société et mentalités collectives en Russie sous l'Ancien Régime, Paris, Institut d'études slaves, 1991, p. 388-421), s'est livré à une nouvelle réflexion sur cette question, ainsi qu'à un commentaire bibliographique. Nous ne citerons évidemment pas les travaux très abondants qui portent sur les institutions académiques ou universitaires, les milieux intellectuels et les individus. Parmi les plus récents, l'ouvrage consacré à l'université de Moscou Universitet dlja Rossii (Une université pour la Russie), t. 1, V. V. Ponomareva et L. B. Horošilova, eds, Vzgljad na istoriju kul'tury XVIII stoletija (Un regard sur l'histoire de la culture du XVIII siècle), Moscou, Russkoe slovo, 1997, offre un aperçu des milieux intellectuels qui se sont constitués autour de l'université de Moscou.
} 
France des années 1880 , dès avant l'affaire Dreyfus ${ }^{2}$, et une autre, qui se concentre sur les milieux universitaires, para-universitaires, académiques depuis le Moyen Âge. Observons que les Temps Modernes et surtout le XVIII ${ }^{\mathrm{e}}$ siècle font souvent figure de transition, soit qu'on garde la même notion en la rendant plus floue car on l'étend à d'autres milieux qu'universitaires, soit qu'on utilise la notion de « protointellectuels » ou d' « intellectuels avant les intellectuels », faisant de cette période, si importante pourtant, une sorte d'antichambre des XIX ${ }^{\mathrm{e}}$ et $\mathrm{XX}^{\mathrm{e}}$ siècles $^{3}$.

C'est la seconde acception que nous adopterons dans l'étude présente afin de l'appliquer au cas russe. Or, comme on le sait, les institutions universitaires et académiques étaient très rares dans la Russie du XVIII ${ }^{\mathrm{e}}$ siècle et se limitaient à l'Académie des sciences avec son université (Saint-Pétersbourg) et à l'université de Moscou, auxquelles s'ajoutaient les institutions médicales. La formation des spécialistes, essentiellement juristes et médecins, qui devaient répondre aux besoins civils de l'État, de même que celle des enseignants universitaires et académiques, se déroulait au moins en partie dans les universités occidentales ${ }^{4}$. Ces séjours laissèrent en Russie des traces diverses, généralement peu abondantes, localisées pour la plupart dans les archives de l'Académie des sciences, du collège des

2. Nous faisons allusion à l'ouvrage de Christophe Charle, Naissance des « intellectuels », 1880 1900, Paris, Éd. de Minuit, 1990, qu'il a depuis prolongé par des essais d'études comparées des intellectuels européens ; voir notamment : id. Les intellectuels en Europe au XIX siècle. Essai d'histoire comparée, Paris, Le Seuil, 1996. De telles études comparées ont été menées en France et ailleurs, souvent collectivement. Voir, par exemple, Michel Trebitsch et Marie-Christine Granjon, eds, Pour une histoire comparée des intellectuels, Bruxelles, Éd. Complexe, 1998 ; Christophe Charle, «Intellectuels, Bildungsbürgertum et professions au XIXe siècle, essai de bilan historiographique comparé (France-Allemagne) », Actes de la Recherche en Sciences Sociales, 106-107, mars 1995, p. 80-95 ; Jürgen Scriewer, Edwin Keiner et Christophe Charle, Sozialer Raum und akademische Kulturen / À la recherche de l'espace universitaire européen, Francfort - Berne, Peter Lang, 1993 ; Wolf Lepenies, Aufstieg und Fall der Intellektuellen in Europa, Francfort - Paris, Campus Verlag - Éd. de la MSH, 1992.

3. C'est dans le livre de Jacques Le Goff, Les intellectuels au Moyen Âge (Paris, Le Seuil, 1957), que, pour la première fois, le terme a été appliqué dans toute sa netteté à la période médiévale. Concernant les Temps Modernes, on peut citer Wilhelmus Frijhof, La société néerlandaise et ses gradués, 1575-1814. Une recherche sérielle sur le statut des intellectuels à partir des registres universitaires, Amsterdam, APA Holland Univ. Press, 1981 ; Roger Hahn, L'anatomie d'une institution scientifique. L'Académie des Sciences de Paris, 1660-1803, YverdonBruxelles-Paris, Éd. des archives contemporaines, s.d., (notamment le chapitre II) ; Didier Masseau, L'invention de l'intellectuel dans l'Europe du XVIII siècle, Paris, PUF, 1994, ainsi que les ouvrages de Robert Mandrou, Françoise Waquet, Hans Bots, Daniel Roche, Robert Darnton, etc.

4. Plusieurs études ont été consacrées à ce sujet . Citons notamment : Erik Amburger, « Die russischen Studenten an deutschen Universitäten bis zur Ende des 18. Jahrhunderts », in id., Beiträge zur Geschichte der deutsch-russischen kulturellen Beziehungen, Geissen, Wilhelm Schmitz Verlag, 1961,p. 214-232 ; Max Okenfuss, «Russian students in Europe in the age of Peter the Great » in John Garrard, ed., The eighteenth century in Russia, Oxford, Oxford University Press, 1973, p. 131-145; Nicholas Hans, «Russian students at Leyden in the 18th century », Slavonic and East European Review, 35, 1957 ; Jürgen Voss, "Les étudiants de l'Empire russe à l'université de Strasbourg au XVIII' siècle », in K. Grau, S. Karp et J. Voss, eds, Deutsch-russische Beziehungen in 18. Jahrhundert, Kultur, Wissenschaft une Diplomatie,Wiesbaden, Harrassowitz, 1997, p. 351-371. Ce sujet est abordé dans les ouvrages ou recueils plus généraux d'E. Winter, Die Geschichte der deutsch-russischen Wissenschaftsbeziehungen im 18. Jahrhundert, Berlin, 1962 et d'Anthony G. Cross, By the bank of Thames, Newtonville (Mass.), Oriental research partners, 1980. 
Affaires étrangères, du collège de Médecine et, pour autant qu'elles n'ont pas brûlé en 1812, de l'université de Moscou. Le plus souvent, il ne s'agit que de quelques mentions dans les documents administratifs ou, dans le meilleur des cas, des rapports qui étaient envoyés par les étudiants à leurs autorités de tutelle en Russie. À ces documents, qui portaient évidemment un caractère officiel, s'ajoutent les matricules des universités occidentales concernées. Autant dire que des sources plus personnelles concernant les étudiants russes à l'étranger - correspondances privées, journaux intimes ou, à défaut, mémoires - sont une denrée assez rare, et par conséquent d'autant plus précieuse lorsqu'on a la chance d'en disposer. Or ce type de documentation permettrait, mieux que toute autre, de comprendre les trajectoires de ces obscurs missionnaires de l'État qui embrassaient des métiers intellectuels et qui étaient si peu nombreux qu'on peut difficilement parler de « milieu » universitaire dans cet air raréfié qu'ils respiraient en Russie. D'autre part, leurs séjours à l'étranger ou, à défaut, leur contact livresque et studieux avec les traditions universitaires européennes leur faisait connaître - le rappeler relève de la banalité - un monde plus ouvert, plus dense, plus cultivé que le leur, ce qui pouvait les conduire à de vastes ambitions et, partant, à des distorsions, des déceptions, des tensions, particulièrement révélatrices comme toute situation conflictuelle, pour peu qu'on en retrouve les traces.

Le cas d'Aleksej Jakovlevič Polenov (1738-1816), qui séjourna à Strasbourg, puis à Göttingen de 1762 à 1767, nous paraît à cet égard particulièrement intéressant. Il est loin d'être inconnu : son petit-fils et érudit Dmitrij Vasil'evič Polenov lui consacra un article dans la revue Russkij arhiv ${ }^{5}$ dans lequel, pour l'essentiel, il relatait le voyage de Polenov à Strasbourg et citait presque in extenso la correspondance de son aïeul dont il disposait. Il publia, la même année et dans la même revue, la réponse d'Aleksej Polenov au fameux concours de la Société libre d'économie en 1766 concernant l'accès des paysans à la propriété foncière ${ }^{6}$. De son côté, M. Šugurov ajouta quelques commentaires sur la situation qui régnait à la direction de l'Académie des sciences avant le départ de Polenov pour Strasbourg, notamment sur les conflits qui opposaient Lomonosov d'une part, à Müller (en Russie, Miller) et Taubert d'autre part ${ }^{7}$. Dans cette étude, nous nous concentrerons sur le séjour de

5. D. V. Polenov « A. Ja. Polenov, Russkij zakonoved» (A. Ja Polenov, juriste russe), Russkij arhiv, 1865, col. 557-614.

6. Ibid., col. 509-540.

7. M. Šugurov, «Učenie i učeniki v XVIII v. » (Les études et les étudiants au XVIII ${ }^{\mathrm{e}}$ siècle), Russkij arhiv, 3, 1866, col. 304-324. Il faut ajouter à ces deux publications la notice consacrée à A. Ja. Polenov et publiée dans Russkij biografičeskij slovar' (Dictionnaire biographique russe), 2e édition, volume Plavil ščikov - Primo, Saint-Pétersbourg, Aspekt-Press, Moscou, 1999, p. 467-470, la notice consacrée à Polenov dans le livre d'E. S. Kuljabko, M. V. Lomonosov $i$ učebnaja dejatel'nost' peterburgskoj Akademii Nauk (Lomonosov et les activités pédagogiques de l'Académie des sciences pétersbourgeoise), Moscou-Leningrad, 1962, p. 176-179 et un article de I.S. Bak, «A.Ja. Polenov, filosofskie, obščestvenno-političeskie i ekonomičeskie vzgliady » (Polenov, ses idées philosophiques, politiques et économiques), Istoričeskie zapiski, 28, 1949, p. 182-202 (étude qui porte sur le projet de Polenov au concours de la Société d'économie) ; voir également les travaux qui concernent l'histoire de l'Académie des sciences, ainsi que Lepehin et Protasov, compagnons de Polenov à Strasbourg : P. Pekarskij, Istorija Imperatorskoj 
Polenov à Strasbourg et, par conséquent, sur les correspondances s'y rapportant, conservées aux archives de l'Académie des sciences et à la Bibliothèque nationale de Saint-Pétersbourg ${ }^{8}$, qui furent déjà assez largement utilisées dans les publications citées, mais sur lesquelles nous espérons jeter un regard nouveau.

Le 13 août (a.s.) 1762, Aleksej Polenov, alors âgé de 23 ans, était employé à l'Académie des sciences dans la fonction de traducteur (il avait reçu ce grade le 31 octobre 1761$)^{9}$ et suivait d'autre part des cours de droit à l'Université académique afin de recevoir une formation de juriste ${ }^{10}$; ce jour-là, il adressa un rapport au secrétariat (kanceljarija) de l'Académie des sciences, dont certains passages méritent d'être cités :

«Depuis que je fus promu au rang de traducteur, je reçus l'ordre du secrétariat de m'employer exclusivement à traduire le droit suédois pour le collège de Justice ainsi que de fréquenter les cours de droit de M. le professeur Fedorovič. Quant aux traductions, comme le secrétariat ne l'ignore pas, j'en ai déjà réalisé assez ; mais jusqu'à présent, elles traînent sans avoir été corrigées : il apparaît ainsi que mon travail et le temps que j'y ai mis ont été vains et qu'il en sera inévitablement ainsi si je demeure dans cet emploi. De la même manière, les cours de monsieur Fedorovič ne sauraient m'être d'une quelconque utilité. Le secrétariat de l'Académie des sciences a décidé de m'y envoyer conformément à mon propre désir et à mon bon vouloir, afin que par la suite, grâce à mes succès, je tire

akademii nauk v Sankt-Peterburge (Histoire de l'Académie impériale des sciences à SaintPétersbourg), vol. 1, Saint-Pétersbourg, 1870, M. I. Suhomlinov, « Istorija rossijskoj akademii » (Histoire de l'Académie de Russie), Sbornik ORJaS, XI, 2, 1874, p. 1-427 ; T.A. Lukina, A. N. Protasov, Russkij akademik XVIII veka (A. N. Protasov, académicien russe du XVIII siècle), Moscou-Leningrad, 1962 ; id., Iv. Iv. Lepehin, Moscou-Leningrad, 1965 ; et enfin : D. Stremooukohoff, «Les Russes à Strasbourg au XVIII' siècle », Revue d'Alsace, vol. 81, 1934, p. 321 , article devenu périmé depuis la publication de celui de Jürgen Voss déjà cité.

8. Respectivement aux Archives de l'Académie des sciences à Saint-Pétersbourg (PFA RAN), f. 3 , inv. 1, d. 270 et 308 et au département des manuscrits de la Bibliothèque nationale de Russie à Saint-Pétersbourg (RNB), f. 595 (Polenovy), d. 86, 88,90-93. Les archives de Polenov à la RNB contiennent quelques lettres qu'il avait reçues à Strasbourg et à Göttingen, mais dont il avait brûlé la plus grande partie comme il s'en explique dans une lettre à Protasov du 7 janvier (n.s.) 1767 (RNB, f. 595, d. 90, f. 11v.), les attestations des professeurs et les copies (faites par lui-même) des lettres qu'il adressa au secrétariat de l'Académie et à d'autres personnes, tout cela en russe et en français. Chaque fois que cela a été possible, nous avons naturellement cité les textes originaux des lettres effectivement reçues à l'Académie. D'autre part, nous avons corrigé quelques erreurs de lecture et des modifications mineures apportées par D. V. Polenov dans sa publication. Nous voulons remercier les collaborateurs des Archives de l'Académie des sciences et du département des manuscrits de la Bibliothèque nationale de Russie, pour l'aide qu'ils nous ont prodiguée et sans laquelle ce travail aurait été impossible, ainsi que les participants du séminaire du Centre d'études du monde russe auquel nous avons pu présenter un résumé de ce travail et dont les remarques et les critiques nous ont été d'une grande aide.

9. PFA RAN, f. 3 , inv. 1, d. 472, f. 224-224v., document cité par E. S. Kuljabko, M. V. Lomonosov i učebnaja dejatel'nost' peterburgskoj Akademii Nauk, op. cit., p. 176.

10. Polenov fit ses études au gymnase de l'Académie, où il fut inscrit en 1749, puis à l'université depuis 1754 , où il faisait des études de droit, tout en perfectionnant son latin, jugé insuffisant. Depuis juillet 1761, il avait été affecté sur ordre du Sénat à la traduction de textes juridiques de Suède et de Livonie. E. S. Kuljabko (op. cit.) indique les différents documents des Archives de l'Académie des sciences d'après lesquels on peut reconstituer la carrière de Polenov. 
un profit direct de mes études ; mais j'ignore ce qu'il en sortira. Le dit monsieur le professeur se contente, et a l'intention de continuer à le faire, de dicter à ses auditeurs de pures définitions, qu'on peut aisément, sans le secours d'un guide, trouver dans un dictionnaire, en épargnant son travail et son temps. Les apprendre par cœur, si poussé soit cet exercice, est tout aussi vain. Il est assurément vrai que certaines sciences sont pleines de termes qui leur sont spécialement propres et que, par conséquent, chaque professeur devrait employer à leur explication plusieurs années avant d'entrer en matière, mais cela n'arrive jamais, comme le prouvent les cours des autres professeurs, dont certains, alors même qu'ils ont commencé bien après lui, sont depuis longtemps entrés en matière. [...] Pour cela, je m'adresse au secrétariat de l'Académie des sciences, le priant de bien vouloir changer quelque chose à cet état de fait, afin que je n'emploie pas mon temps en vain, sans aucune utilité, mais que, profitant d'études convenables et véritables, je puisse apporter plus tard les fruits qu'on attend des gens de notre état (sostojanie). $»^{11}$

Polenov se plaignait donc des cours du professeur de droit Georg Friedrich Fedorovič, qui avait été placé à l'Académie par Lomonosov pour remplacer Strube de Piermont que celui-ci avait contribué à évincer ${ }^{12}$, et réclamait qu'on mît un terme à une situation qui lui faisait perdre son temps dans ses études comme dans son travail. Le ton très ferme, presque arrogant, de cette lettre laisse deviner chez son auteur un sentiment de dignité et un souci de sa propre valeur. Cette attitude ne pouvait guère s'expliquer par l'origine sociale de Polenov. S'il était noble, c'était de petite noblesse, son père était un homme du rang, hautbois dans le régiment de la garde Preobraženskij. Produit de l'Académie, Aleksej Polenov ne pouvait devoir son ascension qu'à ses études, et non pas à sa naissance ou à une carrière militaire. D'autre part, son grade de traducteur lui conférait le $13^{\mathrm{e}}$ rang dans la hiérarchie, de sorte qu'il n'était plus un simple étudiant et qu'il méritait quelque égard, mais ce statut était loin de le placer à l'égal des personnages (le secrétaire de l'Académie, les professeurs) auxquels il s'adressait ${ }^{13}$.

Dès lors son attitude pourrait s'expliquer de trois façons.

La première explication serait que Polenov avait un caractère ombrageux et fier, ce que, de fait, il allait démontrer à plusieurs reprises par son comportement.

La seconde serait qu'il bénéficiait de hautes protections et qu'il se sentait assez soutenu pour s'attaquer à un professeur. Son protecteur n'était autre qu'Ivan Ivanovič Taubert, secrétaire de l'Académie, ce qui lui conférait alors presque tous les pouvoirs à la tête de cette institution. En séjournant à l'étranger, Polenov allait

11. PFA RAN,f. 3 , inv. 1, d. 270, lettre du 13 août 1762, f. 32-32v.

12. À ce sujet, voir M. Šugurov, « Učenie i učeniki v XVIII v. », art. cit, col. 312-315. Strube avait été exclu du corps professoral en 1757 ; voir Protokoly zasedanij konferencii Imperatorskoj Akademii Nauk s 1725 po 1803 (Procès-verbaux des réunions de la conférence de l'Académie impériale des sciences de 1725 à 1803), vol. II (1744-1770), Saint-Pétersbourg, 1899, p. 391. Fedorovič fut nommé en 1760 (ibid., p. 446).

13. Même si ces deux éléments, son appartenance à la noblesse d'une part, son grade de traducteur d'autre part, ne permettent pas de « lire » Polenov, dont les valeurs, comme on le verra, étaient tout autres, il est très possible qu'ils l'aient malgré tout conforté dans sa fierté. 
doubler les rapports officiels qu'il envoyait à l'Académie par des lettres plus personnelles adressées à Taubert. Il le qualifiait souvent de «bienfaiteur » (blagodetel $\left.{ }^{\prime}\right)$ et de «protecteur » (pokrovitel $)^{14}$. Dans une lettre qu'il adressa de Strasbourg à son ami Sauttersheim en octobre 1766 à un moment où, comme on le verra, il se trouvait en conflit avec l'Académie, il évoqua Taubert en ces termes : « [...] j'aurai tort de me méfier d'un homme, dont j'ai eu tant de preuves de sa fermeté et à qui j'ai bien des obligations. $\gg^{15}$ Différentes études, notamment celle de Šugurov et de Kuljabko déjà citées, s'appuient aussi sur l'hypothèse, très plausible, d'une utilisation de Polenov contre Lomonosov ${ }^{16}$. Dans le conflit qui opposait Taubert à ce dernier à la direction de l'Académie et dont celle-ci était l'enjeu, Taubert aurait avancé son «pion » (Polenov) contre Fedorovič, protégé de Lomonosov, façon de prouver que l'université ne remplissait pas son office. De son côté, Polenov se sentait certainement soutenu par Taubert, car celui-ci nourrissait visiblement de grandes ambitions par sa personne interposée. Le moment que nous évoquons coïncida en effet avec une période de codification (plus exactement de tentatives de codification) du droit russe ${ }^{17}$. Avant son expérience malheureuse avec Fedorovič, Polenov avait suivi de 1754 à 1757 les cours de Friedrich-Heinrich Strube de Piermont. Académicien de 1738 à cette dernière date, Strube avait été le premier à tenter une histoire du droit russe ${ }^{18}$, il avait participé en 1754 à la commission sénatoriale chargée d'examiner la législation existante et prononcé en 1756 un Discours sur l'origine et les changemens des loix russiennes ${ }^{19}$. Or, Taubert semblait préparer Polenov à un grand avenir dans la codification du droit russe, dessein qui apparut de plus en plus nettement dans la correspondance entre les deux hommes, surtout en 1766 , à l'heure où se préparait la Commission législative de Catherine. Au cours du séjour de son protégé à l'étranger, il le chargea de préparer un travail écrit sur l'histoire du droit russe, ce dont Polenov lui rendit régulièrement compte dans plusieurs lettres.

14. Par exemple, dans sa lettre du 27 juillet 1763 , RNB, f. 595, d. 90 , f. $1 v$.

15. Ibid., f. 11v. En français dans le texte.

16. Voir notamment M. Šugurov, "Učenie i učeniki v XVIII v. », art. cit., col. 312-315 et E. S. Kuljabko, M. V. Lomonosov i učebnaja dejatel' nost' peterburgskoj Akademii Nauk, op. cit., p. 177, qui s'appuie sur une réflexion de Lomonosov pour soutenir cette thèse P. S. Biljarskij, Materialy dlja biografii Lomonosova (Matériaux pour une biographie de Lomonosov), Saint-Pétersbourg, Académie des sciences, 1865, p. 081, formule la même analyse au sujet de l'envoi de Protasov à l'étranger en 1751.

17. À ce sujet, voir V. N. Latkin, Zakonodatel'nye komissii v Rossii $v$ XVIII st. Istorikojuridičeskoe issledovanie (Les commissions législatives en Russie au XVIII siècle. Étude d'histoire juridique), Saint-Pétersbourg, 1887.

18. Voir par exemple le manuscrit en français, resté inédit (anonyme, mais attribué à Strube), consacré à la Russkaja pravda, « Loi Russienne donnée aux habitants de Novgorod par le grand duc Jaroslaf et ses fils en 1017 » (Bibliothèque de l'Académie des sciences, Saint-Pétersbourg, Département des manuscrits, cote $\mathrm{F} \mathrm{n}^{\circ} 230$ ) dont le préambule est une esquisse d'histoire du droit russe. Sur Strube, voir Russkij biografičeskij slovar', op. cit., vol. Smolovskij Suvorina, p. 547-549.

19. Publié en russe et en français dans le même volume, à l'imprimerie de l'Académie des sciences, Saint-Pétersbourg, 1756. 
La troisième explication du comportement de Polenov était qu'il se sentait appartenir à une élite, non pas à la noblesse mais à la catégorie des spécialistes instruits, qu'il était pénétré du sentiment de sa propre dignité, ou plus exactement de l'importance, du sérieux, de l'utilité de sa double activité - travail de traducteur d'une part et études d'autre part - et qu'il en défendait la bonne marche en «professionnel » possédant un savoir et sachant comment l'acquérir. Symptomatique était également son insistance sur son propre bon vouloir, sa démarche individuelle s'inscrivant à la fois dans une activité intellectuelle, qui lui donnait son sens, et dans un milieu professionnel donné, celui de l'Académie, car c'est ainsi qu'il faut comprendre selon nous son allusion finale à son « état».

Nous ne reviendrons plus sur la première explication, qui ne présente pas d'intérêt historique, passerons rapidement sur la seconde qui, si intéressante qu'elle soit, ne répond pas aux questions qui nous intéressent, pour nous concentrer sur la troisième. En effet, les deux premières explications, sans doute vraies toutes les deux, n'empêchent pas que les termes dont usait Polenov, les valeurs auxquelles il se référait, relevaient d'un sentiment de soi qui le classait selon nous dans la catégorie des « intellectuels».

La démarche de Polenov prend encore plus de sens si nous la replaçons dans un contexte plus large que celui de son propre cas. Sa plainte coïncidait en effet, jour pour jour, avec une demande d'Aleksej Protasov, professeur adjoint (ad'junkt) de l'Université académique, qui, en des termes beaucoup plus respectueux que Polenov (alors qu'il lui était supérieur dans sa fonction, assistant de droit aux réunions académiques), demandait à se rendre à Strasbourg afin de parfaire son instruction déjà acquise à Leyde et à Strasbourg, et d'y recevoir le titre de docteur ${ }^{20}$. Mieux encore, trois autres étudiants demandèrent au même moment de séjourner dans des universités étrangères; il s'agissait d'Ivan Ivanovič Lepehin, qui avait alors vingt et un ans et qui allait faire la carrière que l'on sait (c'était le futur voyageur sibérien et le traducteur de Buffon), de Gerasim Špynev et de Nikolaj Strešnev ${ }^{21}$. Le ton et la forme de ces requêtes étaient stéréotypés, à telle enseigne que certains passages semblent avoir été copiés mot pour mot à partir d'une même source, voire à la suite d'une même instigation. Cette coöncidence ne pouvait être fortuite et s'expliquait peut-être par l'hypothèse, déjà mentionnée, d'une intrigue ourdie contre Lomonosov et qui tendait à dégarnir, tout en la dépréciant, l'université qu'il dirigeait.

20. Lettre du 13 août 1762, PFA RAN, f. 3, inv. 1, d. 270, f. 30-31. Protasov avait été envoyé à l'université de Leyde en 1751 pour y faire des études de médecine, où il demeura jusqu'en 1755. De 1755 à 1757 il poursuivit ses études à Strasbourg, puis de nouveau à Leyde jusqu'en 1759 , après quoi il retourna à Saint-Pétersbourg. Au sujet de ses voyages, voir l'ouvrage de T. A. Lukina, A. N. Protasov, Russkij akademik XVIII veka, op. cit., p. 33-54.

21. PFA RAN, f. 3, inv. 1, d. 270, respectivement f. 33-33v., 34-34v. et 35. 
Mais là encore, l'intrigue n'explique pas tout, et notamment pas les arguments avancés par les étudiants à l'appui de leur demande de séjour à l'étranger. Citons la lettre de Lepehin qui était la première du lot :

«En vertu de l'ordre (prikaz) donné au début de cette année 1762 par son honneur monsieur le conseiller de collège Lomonosov, selon lequel les étudiants de céans devaient s'appliquer à une seule science selon leur désir et leur capacité, moi, sous-nommé, bien que je me sois senti depuis longtemps une inclination particulière pour l'histoire naturelle, je ne pouvais m'y consacrer en l'absence d'un professeur capable de m'y instruire ; cependant, dans l'attente d'une occasion de m'appliquer exclusivement à cette science et sachant que la chimie allait grandement contribuer et était même tout à fait indispensable à mes succès futurs en histoire naturelle, je me suis engagé à présent dans cette science auprès de monsieur [1'académicien] Lehmann et je m'y emploie aujourd'hui. Et comme beaucoup [d'étudiants] de l'université de Moscou et de sa faculté de médecine comme de l'Académie des sciences furent envoyés en contrées étrangères aux frais de Sa Majesté Impériale pour qu'ils y apprennent les sciences afin de hâter la diffusion des sciences parmi les sujets natifs de l'empire de Russie, inspiré par ces exemples je prie très humblement le secrétariat de l'Académie des sciences qu'il veuille bien, suivant l'exemple de monsieur le professeur Kotel'nikov, des professeurs adjoints Protasov et Rumovskij, m'envoyer aussi, humble serviteur que je suis, dans l'école ${ }^{22}$ étrangère qu'il jugera bon de choisir, afin que je m'y instruise en histoire naturelle déjà mentionnée, selon mon inclination. $»^{23}$

On retiendra de ce texte la référence aux caractéristiques individuelles (inclination et capacités) de l'étudiant, l'importance accordée à son parcours personnel, l'allusion à la diffusion des sciences parmi les Russes de souche - cheval de bataille favori de Lomonosov —, enfin la mention des quelques « Russes » qui avaient déjà suivi la même voie. L'ordre de Lomonosov ${ }^{24}$ aurait donc servi de prétexte à plusieurs démarches individuelles, qui rejoignaient celle de Polenov et qui faisaient toutes appel à une conception libérale, au sens ancien et universitaire du mot, des études, tant sur la question du choix de la matière étudiée que du lieu des études, même si, sous ce dernier rapport, la demande restait vague.

Le réponse de l'Académie fut exceptionnellement rapide au regard des lenteurs bureaucratiques habituelles, ce qui tendrait à montrer, soit que Müller et Taubert étaient déjà au courant de ces démarches, voire qu'ils les avaient encouragées, soit qu'ils s'en saisirent activement parce qu'ils y étaient favorables. Le 19 août, Müller adressa un rapport à l'Académie, dans lequel il traitait les cinq cas. Il proposait de satisfaire la demande de Protasov, d'envoyer Polenov dans une université alle-

22. Les deux autres étudiants usent des termes universitet ou akademija, à l'époque synonymes.

23. Lettre du 13 août 1762, ibid., f. 33-33v., reproduite également, à quelques modifications minimes près, dans M. I. Suhomlinov, Istorija rossijskoj akademii, op. cit., p. 168-169.

24. Nous n'en avons jusqu'à présent trouvé aucune trace dans les sources disponibles. Il se peut, même si c'est peu probable, que cet ordre eût été donné oralement. Par ailleurs, une telle orientation eût été parfaitement conforme aux idées de Lomonosov sur l'organisation des études à l'université. 
mande (« il ne serait pas mauvais de l'envoyer dans une académie allemande [...] selon son désir ») et Lepehin à Uppsala auprès de Linné ou bien dans quelque autre université en Allemagne ; quant aux deux autres requêtes, il convenait de les laisser pour le moment sans suite, car ces étudiants n'avaient pas encore atteint le niveau requis pour profiter d'un séjour à l'étranger ${ }^{25}$. Le 26 août, un rapport de Taubert adressé à Kirill Razumovskij lui faisait part des conclusions de la conférence académique, qui (en l'absence de Lomonosov, précisons-le) reprenait les mêmes propositions, avec cette précision que Polenov devait se rendre à l'université de Leipzig $^{26}$.

Enfin, le 3 septembre, un rapport de Müller exposait les conclusions que la conférence avait adoptées la veille ${ }^{27}$; cette fois, on décidait d'envoyer Polenov, Lepehin et Protasov à l'université de Strasbourg pour des raisons qu'il est intéressant de mentionner : le théâtre de la guerre de Sept ans rendait la Saxe dangereuse, tandis que Strasbourg était en paix ; la vie, sans doute pour les mêmes raisons, y serait moins chère ; les deux étudiants seraient accompagnés par Protasov, qui allait y séjourner de toute façon. Enfin, ils seraient surveillés (nadzirat') à Strasbourg par le professeur d'histoire et de rhétorique Jean-Daniel Schöpflin, intellectuel de renommée européenne, courtisé par les cours et les académies, dont celle de Russie ( il en était du reste membre d'honneur depuis 1740). Lepehin aurait droit également à la tutelle du chimiste et botaniste Jacob Spielmann, auquel Lehmann avait promis d'écrire. Le même texte esquissait le programme d'études des deux jeunes gens : ils devaient apprendre d'abord les humanités (gumaniory), l'allemand et le français, après quoi seulement ils attaqueraient leurs études spécialisées ; Polenov, en particulier, devrait «apprendre les antiquités [drevnosti], l'histoire du droit [istorija jurisprudencii] et le droit de la nature et des gens [natural'noe $i$ obščenarodnoe pravo] » avant d'aborder les études de droit proprement dites ${ }^{28}$.

Les instructions ${ }^{29}$ dont, comme c'était l'usage, on allait doter les étudiants en partance, reprenaient en gros ces différentes dispositions, sans rien ajouter au rapport de Müller concernant les plans d'études. Elles étaient rédigées dans un style stéréotypé (il s'agissait du même texte, à quelques mots près, simplement adapté à la spécialité de chaque étudiant) et quasi militaire qui prévalait, et allait continuer

25. PFA RAN, f. 3, inv. 1, d. 270, f. 36-37.

26. Ibid., f. 38-38 v. Voir également Protokoly zasedanij konferencii Imperatorskoj Akademii Nauk s 1725 po 1803, vol. II, op. cit., p. 485-86. La date indiquée dans cet ouvrage (9 août 1762) paraît erronée.

27. Voir ces conclusions, plus succinctes que le rapport de Müller, ibid., p. 488. Cette fois Lomonosov, mais aussi Taubert, furent absents de la réunion.

28. Ibid., f. 44-44 v.; on trouve aussi une copie de ce texte dans les archives de l'académicien Jacob Stählin (RNB, f. 871, d. 116). Spielmann allait devenir membre d'honneur de l'Académie le 14 avril 1763 .

29. Voir, en ce qui concerne Polenov, l'ukaz impérial n 1049 du 3 septembre (RNB, f. 595, d. 86, f. 1), l'instruction reçue et signée par lui (f. 2-2 v.) ou son brouillon aux archives de l'Académie (PFA RAN, f. 3, inv. 1, d. 270, f. 48-48 v.), dont les ratures indiquent une hésitation entre Leipzig et Strasbourg. Pour Lepehin, voir ibid., f. 50-51, ou M. I. Suhomlinov, Istorija rossijskoj akademii, op. cit., p. 170-171. 
de prévaloir, dans ce type de documents. Citons seulement deux passages caractéristiques du texte destiné à Polenov :

«1. Tu dois d'abord craindre Dieu et garder intacte la foi gréco-russe dans toute la mesure du possible et en outre, gardant l'honneur de l'Académie qui t'a envoyé poursuivre tes études, tu dois avoir une vie respectable et constante, et fuir tous les vices, surtout l'ivrognerie qui est la cause de tous les maux. [...]

4. De Strasbourg, tu devras envoyer au secrétariat des rapports sur tes succès, accompagnés des attestations de messieurs les professeurs de là-bas et ne pas revenir ici sans l'ordre du secrétariat. »30

Rien de surprenant dans ce texte, écrit sur le mode d'un ukaz impérial (d'où le tutoiement, alors que Taubert vouvoyait Polenov), et semblable aux instructions rédigées précédemment, par exemple celle qui avait été adressée à Protasov en 1751 en des termes quasi identiques ${ }^{31}$. Mais voici le paragraphe 5 qui, lui, à notre connaissance, était parfaitement original :

«Lorsqu'il te semblera que tu as assez vécu à Strasbourg et que tu voudras aller dans une autre académie, tu devras en rapporter en temps voulu au secrétariat et attendre sa décision, et ne partir de ton propre chef sous aucun prétexte. $\gg^{32}$

Cette disposition libérale, particulièrement surprenante sur le fond général de ce texte, nous paraît relever du même esprit universitaire (ou académique) qui avait présidé aux démarches des jeunes gens ${ }^{33}$. Comme on va le voir, elle ne resta pas lettre morte aux yeux de Polenov, qui allait se comporter comme si elle allait de soi.

Revenons à présent sur le choix de Strasbourg. Ainsi qu'on a pu le constater, les raisons qui y avaient conduit, sans que la documentation existante ne nous permette du reste d'attribuer cette décision à une personne précise, étaient conjoncturelles et ne tenaient en rien à la spécificité de cette université, si ce n'était son bilinguisme, qui s'ajoutait évidemment à l'étude du latin et auquel le rapport de Müller faisait implicitement allusion. Polenov aurait pu s'initier aux matières citées dans la plupart des universités allemandes, où le droit naturel était enseigné depuis la fin du XVII siècle, par exemple à Heidelberg, Halle, Leipzig, etc., et où les doctrines de Grotius, Pufendorf, Thomasius et Wolff étaient très influentes. Sans la guerre, Polenov serait probablement allé à l'université de Leipzig ou de Tübingen, fréquemment citées dans les correspondances de l'Académie ${ }^{34}$.

Le choix de Strasbourg faisait figure de nouveauté. Auparavant, les quelques étudiants qui avaient été envoyés par les différentes institutions russes, étaient surtout allés à Leyde, Königsberg, Halle, Marburg, Leipzig, Uppsala. Au moment

30. RNB, f. 595, d. 86, f. 2-2v.

31. Voir le texte de cette instruction dans PFA RAN, f. 3, inv. 1, doc. 804, f. 1, cité par T. A. Lukina, A. N. Protasov, Russkij akademik XVIII veka, op. cit., p. 31-32.

32. RNB, f. 595 , d. 86 , f. $2 \mathrm{v}$.

33. Elle s'explique peut-être aussi par l'antécédent de Protasov qui était passé de Leyde à Strasbourg.

34. Voir par exemple, une lettre de Müller datée du 18 mars 1757, PFA RAN, f. 21, inv. 3, d. $307 /$

17, citée dans T. A. Lukina, A. N. Protasov, Russkij akademik XVIII veka, op. cit., p. 47. 
que nous évoquons, plusieurs d'entre eux - peut-être une dizaine - séjournaient à l'étranger ${ }^{35}$. Toutefois, Strasbourg n'était pas une terre inconnue. Polenov et Lepehin y avaient été précédés par d'autres sujets de l'Empire depuis l'époque de Pierre le Grand ; il s'agissait, presque toujours, d'Allemands baltes et de quelques Ukrainiens. Plus proches des cas qui nous occupent, il faut citer, comme on l'a vu, Protasov, de 1755 à 1757, l'étudiant en médecine Savva Leontovič, inscrit depuis 1761 , les deux frères Grigorij et Ivan Šein, inscrits à la faculté de philosophie et que Polenov évoqua dans plusieurs de ses lettres. Par la suite, les séjours d'étudiants russes allaient s'y succéder, de sorte que pendant longtemps cette université allait faire partie des plus fréquentées par les Russes, soit 138 étudiants au cours du $\mathrm{XVIII}^{\mathrm{e}}$ siècle ${ }^{36}$. Signalons enfin qu'au cours du séjour de Polenov à Strasbourg, il vit arriver en 1765 un nommé Dimitrij Legkoj, également étudiant en droit $^{37}$, ainsi que Petr et Andrej Razumovskij, les deux fils de Kirill, à l'époque président de l'Académie des sciences, qui allait lui-même visiter Strasbourg en $1766^{38}$.

Dès le début des années 1760, ces Russes strasbourgeois se partageaient ainsi entre les enfants de la haute noblesse, accueillis par Schöpflin à l'École diplomatique qu'il avait fondée vers 1752, et les étudiants en médecine et en droit dont il s'occupait moins, mais sur lesquels il veillait néanmoins personnellement.

Or Polenov allait bénéficier d'un enseignement spécifique, qui était en passe de faire de l'université de Strasbourg une des meilleures et des plus prisées d'Europe ${ }^{39}$.

35. Le décompte exact des séjours d'études des Russes à l'étranger est loin d'être facile, parce que la documentation les concernant est dispersée entre les archives des différentes institutions; d'autre part, les archives diplomatiques et consulaires russes ne fournissent pas d'informations exhaustives (même celles de la délivrance des passeports); enfin les registres des matricules des différentes universités ne sont jamais complets. Signalons un relevé de ces étudiants originaires de la seule université de Moscou, commandé par Ivan Šuvalov en 1763 et effectué au collège des Affaires étrangères. On y trouve Ven'jamin Zabelin en médecine à Königsberg depuis 1759, Ivan Tret’jakov et Semen Desnickij à Glasgow depuis 1761 et enfin Aleksandr Karamyšev et Matfej Afonin en histoire naturelle à Uppsala, sous la direction de Linné : voir la lettre d'Ivan Melissino du 9 avril 1763, Archives de la politique étrangère de l'Empire de Russie (AVPRI), Moscou,f. 2 (Vnutrennie kolležskie dela), inv. 6, d. 1750, f. 1-1v.

36. Voir J. Voss, «Les étudiants de l'Empire russe à l'université de Strasbourg au XVIII ${ }^{\mathrm{e}}$ siècle », art. cit., p. 357 ; ce chiffre rectifie celui avancé par D. Stremooukohoff, « Les

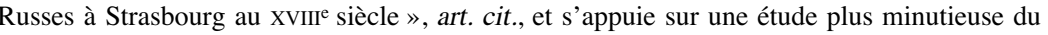
matricule de Strasbourg publié par ailleurs par Gustav C. Knod (Die Alten Matrikeln der Universität Strassburg 1621-1793, 3 vol., Strasbourg, Karl Trübner, 1897).

37. PFA RAN, f. 3 , inv. 1 , d. 308, f. 120

38. Voir la lettre de Polenov à Protasov du 20 ou 23 mai 1765, RNB, f. 595, d. 90, f. 6v. : Legkoj arriva à Strasbourg le 14 mai, et accompagnait les Razumovskij.

39. Au sujet de l'université de Strasbourg, de l'enseignement de l'histoire, de Schöpflin et de ses élèves Lorenz et Koch, on peut consulter les études suivantes : Georges Livet, «L'université de Strasbourg et son rayonnement européen au Siècle des Lumières ", Revue des Sciences Sociales de la France de 1'Est, 1989-1990, p. 50-66 ; Bernard Vogler et Jürgen Voss, eds, Strasbourg, Schöpflin et l'Europe au XVIII' siècle, Bonn, Bouvier Verlag, 1996 ; Jürgen Voss, Jean-Daniel Schöpflin (1694-1771). Un Alsacien en l'Europe des Lumières, Strasbourg, Société savante d'Alsace, 1999 ; Georges Livet, « L'enseignement de l'histoire à l'Université de Strasbourg aux XVII et XVIII ${ }^{e}$ siècles », Bulletin de la Société d'Histoire du Protestantisme français, vol. 135, janvier-février-mars, 1989, p. 117-141 ; A. Salomon, « Jean-Michel Lorenz, professeur d'éloquence à l'Académie de Strasbourg, 1723-1801) », Revue d'Alsace, 71, 1924, p. 444-467, et 72, 1925, p. 48-61, 143-152. 
Pour être bref, nous dirions que le système mis au point par Schöpflin associait intimement l'enseignement du droit et de l'histoire, ce qui avait pour conséquences la modernisation de la première discipline et l'anoblissement de la seconde. Certes le droit «moderne », fondé sur les ordonnances royales, était déjà enseigné dans les universités françaises de sorte que la formation du juriste ne s'y limitait plus comme autrefois au droit romain et au droit canon ou ecclésiastique, et d'autre part, les universités allemandes avaient déjà instauré l'étude du droit naturel. Mais le système strasbourgeois allait beaucoup plus loin. Les études juridiques comprenaient désormais celle du droit international, c'est-à-dire des traités, ainsi que la recherche des sources historiques des droits nationaux, notamment germanique. Quant à l'histoire, elle était enseignée sur la base des sources diplomatiques. Ajoutons enfin que cette configuration, tout au moins l'enracinement national et historique du droit, avait pris naissance dans les universités allemandes, mais tout particulièrement à l'université de Göttingen qui, récemment créée, apparaissait comme un véritable phare des Lumières et qui se trouvait en relation étroite avec celle de Strasbourg ${ }^{40}$.

Les débuts du séjour de Polenov ${ }^{41}$ s'avérèrent difficiles. Certes, Schöpflin lui fit bon accueil et lui ouvrit, comme il le faisait souvent avec ses étudiants, sa bibliothèque avec permission d'en user « autant qu'il le voulait "2 $^{4}$. Mais, outre les difficultés qu'il devait éprouver dans l'assimilation du français, largement utilisé à Strasbourg, Polenov ne comprit guère le plan d'études qui lui avait été préparé par Lorenz ${ }^{43}$, professeur extraordinaire, élève et assistant de Schöpflin qu'il remplaçait à la chaire d'éloquence (de rhétorique) et d'histoire ; il se demandait pourquoi il lui fallait étudier ces deux matières qui lui étaient enseignées par le même Lorenz et non aborder plus rapidement l'étude du droit tel qu'il l'imaginait. En décembre 1763 , soit un an après son arrivée, il demanda à Taubert la permission de partir pour Leipzig, ce qui lui fut refusét4.

40. Au sujet de la question de l'enseignement du droit et de l'histoire, voir surtout l'ouvrage de Notker Hammerstein, Jus und Historie. Ein Beitrag zur Geschichte des historischen Denkens an deutschen Universitäten in späten 17. und 18. Jahrhundert, Göttingen, Vandenhoeck \& Ruprecht, 1972, et aussi, concernant la France, Christian Chêne, L'enseignement du droit français en pays de droit écrit (1679-1793), Genève, Droz, 1982.

41. Il arriva avec ses compagnons à Strasbourg le 29 novembre (n.s.) 1762 (voir son rapport à l'Académie du 22 janvier 1763, PFA RAN, f. 3, inv. 1, d. 270, f. 63).

42. Copie de la lettre de Polenov à Taubert du 2 juillet 1763 (RNB,f. 595, d. 90,f. 1v.).

43. On le sait par une lettre de Polenov à Protasov du 17 juillet 1764 (ibid., f. 3v.). Plus tard, Polenov écrit qu'il a choisi ses cours (kollegii) «sur le conseil de mes maîtres » (rapport du 22 avril 1765, PFA RAN, f. 3, inv. 1, d. 270, f. 110)

44. Voir la copie de sa lettre à Taubert du 8 décembre 1763 (RNB, f. 595, d. 90, f. 2v.-3). Nous ne disposons pas de la réponse négative de Taubert, mais une lettre de Polenov d'avril 1764 l'évoque explicitement (PFA RAN, f. 3, inv. 1, d. 270,f. 116). 
Les rapports et les attestations (attestaty) que Polenov adressa au secrétariat de l'Académie nous permettent de reconstituer avec une certaine précision les cours qu'il suivit :

De novembre 1762 à juillet 176445

- Latin, par Jean-Michel Lorenz (langue et style, Annales de Tacite, Virgile, Orationes de Cicéron)

- Rhétorique, par Lorenz

- Histoire, par Lorenz (histoire abrégée du Saint Empire, puis histoire universelle d'après Cellarius)

- Antiquités romaines (mœurs et rites des Romains) par André Lamy, bibliothécaire à Strasbourg

- Métaphysique

De l'été 1764 à l'été $1765^{46}$

- Histoire du Saint Empire, par Lorenz

- Histoire de l'Église, par Lorenz

- Histoire politique (généalogie et origine des principales maisons allemandes actuellement régnantes), par Jean-Daniel Schöpflin

- Histoire des traités de paix, par Schöpflin

- Droit criminel et droit romain, $2^{\mathrm{e}}$ partie, par Johann Reinhard Kugler

- Droit de la nature et des gens, par Friedrich Ehrlen

- Droit public du Saint Empire, par Ehrlen

- Droit romain (première partie), par Ehrlen

- Les Institutiones de Justinien, par Johann Christian Treitlinger

De septembre 1765 à septembre $1766^{47}$

- Droit civil et criminel, par Kugler

- Droit canon, par Kugler

- Histoire des traités de paix, par Schöpflin

- Droit public du Saint Empire, par Ehrlen

- Droit de la nature, par Ehrlen

- Droit romain (première partie), Ehrlen

- Droit national, par Treitlinger

- Les Institutes de Justinien, par Treitlinger

À cette liste s'ajoutent la lecture et les exercices de français et un projet, non réalisé faute de temps, qui était l'apprentissage du grec.

Ce n'est pas notre propos d'analyser ici en détail un programme et des enseignements qui, par ailleurs, offrent bien des aspects intéressants. On en retiendra que Polenov ne commença à apprendre les matières strictement juridiques qu'à partir de la troisième année de son séjour à Strasbourg; encore les disciplines les plus traditionnellement juridiques comme le droit romain, les institutiones, le droit canon ne furent-elles abordées qu'assez tard. Par contre, le droit naturel fut le

45. Ibid., f. 63v., 124, 126-127, 132, 134, 138 ; RNB, f. 595, d. 90, f. 3 v.

46. Ibid., f. 110-114, 119-120, 140, 144, 149 ; RNB, f. 595, d. 90, f. 5v.-6.

47. Ibid., f. 60, 142 ; RNB, f. 595, d. 88, f. 1-3. 
premier à apparaître avec le droit des gens (dans la tradition de Pufendorf, Grotius et Wolff), et surtout l'histoire demeura présente presqu'à chaque pas de l'étudiant car, par exemple, l'histoire ecclésiastique et celle du Saint Empire, enseignées par Lorenz, servirent de propédeutique au droit canon et au droit public, qui étaient de la sorte «historicisés ». De matière auxiliaire ou propédeutique, l'histoire faisait désormais partie des disciplines les plus nobles.

Or tout porte à croire que Polenov, d'abord dérouté par cette organisation des études, ceci d'autant plus que ses mentors russes, formés au début du XVIII siècle, n'en avaient certainement qu'une idée très vague et ne l'y avaient guère préparé, cessa de la subir et l'adopta au point de s'en faire, comme on le verra, un farouche défenseur. À plusieurs reprises dans ses rapports au secrétariat ou dans ses lettres à Taubert, il ne se contenta pas de rendre compte des « collèges » qu'il avait suivis, il en livra la philosophie profonde et fit part de ses intentions qui, naturellement, allaient dans le même sens. En particulier, il répéta à plusieurs reprises que les «arts libéraux » (svobodnye nauki) lui étaient nécessaires pour la carrière à laquelle il se destinait. Dans un rapport à l'Académie daté du 13 septembre 1765, par exemple, il évoque ses travaux assidus en arts libéraux et en langues « lesquels, comme je le sais assez moi-même, sont indispensables à mes intentions. $\gg^{48} \mathrm{Du}$ reste, Schöpflin signifia à plusieurs reprises sa satisfaction devant les progrès de son élève et il en fit part notamment à Kirill Razumovskij quand celui-ci séjourna à Strasbourg ${ }^{49}$.

Cette adaptation intelligente, c'est-à-dire consciente et volontaire, aux conditions strasbourgeoises, apparut de façon quelque peu dramatique au cours d'un conflit qui opposa Polenov au secrétariat de l'Académie. En décembre 1765, Polenov et Lepehin écrivirent conjointement une lettre à Protasov ${ }^{50}$, auquel ils annoncèrent la fin prochaine de leurs études à Strasbourg à Pâques 1766 et leur intention de se rendre dans une autre université afin de perfectionner leurs connaissances et leur pratique des langues vivantes ${ }^{51}$. Bien plus tard, nous apprenons par la correspondance de Polenov avec son ami Sauttersheim, sur lequel nous reviendrons, que Polenov avait l'intention de se rendre à Paris pour des raisons qu'il n'explicita guère ; on sait seulement par sa lettre que l'étudiant Leontovič, déjà cité, et Sauttersheim se trouvaient alors dans la capitale française. Le 24 avril 1766,

48. RNB, f. 595, d. 90,f. 7. Souligné par nous. On notera le ton très personnel de Polenov.

49. Voir la lettre de Polenov à Protasov du 29 mars 1766, RNB, f. 595, d. 90, f. 9 : « il [Razumovskij] fut content de ce que M. Schöpflin lui a dit en notre faveur » (en français dans le texte).

50. Après avoir soutenu son doctorat en juin 1763, Protasov était rentré à Saint-Pétersbourg. Il avait noué des relations d'amitié avec les deux étudiants et leur servit de confident et d'intermédiaire, même si, au cours du conflit qui opposa Polenov et l'Académie en 1766, il s'empressa de se ranger du côté du manche.

51. RNB, f. 595, d. 90, f. 7v.-8. Comme le note M. Šugurov ( Učenie i učeniki v XVIII v. », art. cit., col. 319-322) et comme on le lit clairement dans la lettre, cette démarche se situait dans un climat d'inquiétude, car les deux jeunes gens, probablement informés par Protasov, avaient eu vent des changements qui s'annonçaient à la tête de l'Académie des sciences avec la nomination de Vladimir Grigor'evič Orlov comme directeur, et cherchaient à assurer leur avenir. 
Polenov fit part à celui-ci d'une requête que Schöpflin, à sa demande, aurait adressée à l'Académie pour défendre son projet : « J'ai lu sa lettre et crois en même tems qu'elle produira l'effet que je désire. $\aleph^{52}$ Mais en juillet, Polenov reçut une lettre de Protasov, d'où il ressortait que l'assemblée professorale lui reprochait d'avoir passé trop de temps à faire de l'histoire et des matières « qui ne pourraient que très peu servir » à sa « future profession ». Foin, donc, de généalogie germanique, d'histoire ecclésiastique et de droit de l'Empire ; Polenov devait terminer au plus vite ses études à Strasbourg en se concentrant sur la jurisprudence, à défaut de quoi il devrait les achever dans une autre université allemande, par exemple à Tübingen ${ }^{53}$.

La réaction de Polenov fut violente, comme il le laissa paraître dans ses lettres à Sauttersheim, à Protasov, enfin à l'Académie elle-même. À son ami, il écrit en français :

« J'ai affaire à des gens insensés dont l'entêtement et la stupidité me font enrager. Ces messieurs m'ont fait des propositions malveillantes sans oublier d'y joindre des reproches, si je ne veux pas aller à Tübing pour finir mes études. C'est une étrange disposition qui mérite que je leur réponde en pareils termes et voilà justement ce que je ne manquerai pas de faire. $»^{54}$

Une semaine après, il promet d'adresser aux dits messieurs une réponse « qui fera bien danser les sots animaux dont je vous ai parlé ; ces bêtes ne s'attendent pas à cela, je crois que leur étonnement sera bien grand. Mais ces messieurs n'ont pas mérité qu'on les traite d'une autre façon. Ils m'ont obligé d'être grossier vis-à-vis d'eux. $\gg^{55}$ Et enfin, en septembre :

«Ces messieurs prennent la modestie pour la bassesse. [...] Je les connais trop et je les hais comme les ennemis de ma patrie; la meilleure façon de se tirer d'affaire est celle d'avoir une téméraire hardiesse pour les intimider, alors ces tigres deviennent aussi paisibles que les moutons [...]. Je me prépare d'être malheureux ; l'attachement pour ma patrie en sera la cause, je ne penserai qu'à la servir et voilà justement la source d'où viendra peut-être mon malheur. »56

A Protasov, il répond en août, également en français :

« Après avoir lu votre lettre, je n'ai pu m'empêcher de rire. Il faut avouer, que je ne m'attendais pas à cela et si ces étranges raisonnements viendront à s'éclairer un jour tout le monde en sera fort étonné. Le mécontentement de nos messieurs

52. RNB, f. 595, d. 90, f. 9v. (en français dans le texte). Nous n'avons pas trouvé trace de cette lettre de Schöpflin dans les archives de l'Académie des sciences; l'attitude impatiente de l'Académie à l'égard de Polenov laisse douter qu'elle l'ait reçue.

53. RNB, f. 595, d. 92, f. 1 (lettre du 23 juin 1766).

54. RNB, f. 595, d. 90, f. 10-10v. (lettre du 29 juillet 1766).

55. Ibid., f. 10v. (lettre du 4 août 1766).

56. Ibid., f. 11v. (lettre du 2 septembre 1766). 
les professeurs ne pourrait être si ridicule qu'il ne vaut pas la peine d'en parler davantage $[s i c]$; pourtant comme je suis obligé en quelque façon d'y répondre, je vous dirai qu'il ont bien tort dans ce cas-ci, car tous les collèges, que j'avais fréquenté, sont de la dernière importance pour moi et même, supposons qu'ils ont raison, mais il fallait m'en avertir de bonne heure, pour à présent [sic] quand je viens presque de finir mon cours d'études, tous leurs conseils sont tout à fait inutiles. Dès mon arrivée à Strasbourg jusqu'à ce tems-ci j’étais, pour ainsi dire, abandonné de tout le monde et pour n'avoir rien à me reprocher, je me suis par bonheur pour moi si bien arrangé tant dans ma conduite que dans mes études que tous les Messieurs les Professeurs d'ici en étoient fort contents, aussi c'était à eux que j'avais mon secours dans tout ce qui regardait mes études et leur arrangement et sans les en informer auparavant je n'osais rien entreprendre. C'étoit justement le tems, ou j'avois bien besoin de leurs sages avis ; mais ils gardaient un profond silence : à cette heure-ci je sais très bien moi-même tout ce qu'il me faut. Voilà ma façon de penser qui étoit simple et naturelle, n'offensant personne [...]. »57

Et enfin il adresse à l'Académie une lettre circonstanciée et particulièrement riche, naturellement en russe, dont voici l'extrait le plus significatif :

«J'apprends également que l'assemblée professorale, sans qu'en toute justice, elle ait eu pour cela aucune raison ou prétexte, a fait preuve de son mécontentement à l'égard de ma longue présence aux collèges d'histoire, prétendant qu'ils me serviraient peu dans ma future profession. Je n'ai en rien enfreint l'instruction, bien que manifestement insuffisante, qui me fut donnée, suivant laquelle et sur le conseil de messieurs les professeurs de céans, j'ai organisé mes études, ainsi que mes rapports l'attestent clairement ; ainsi je ne trouve aucune raison qui eût pu les conduire à s'indigner. Je suis très étonné que messieurs les professeurs respectent si peu les arts libéraux, surtout leur partie la plus noble que sont les connaissances historiques, qui servent de fondement essentiel à ma charge. Sans des connaissances suffisantes dans ce domaine, aborder d'emblée le droit serait aussi insensé que de vouloir abattre des arbres avec le manche d'une hache. Qu'on se souvienne seulement de l'exemple probant des grands juristes d'une époque peu favorable aux sciences qui, sans avoir de notion de l'histoire et des antiquités, tombèrent dans une erreur telle qu'on ne peut songer à eux, qui furent au reste si grands, sans éprouver du regret. Qui ne sait qu'autrefois Alciat, Cujas, Hotman, Brisson, Rittershusius ${ }^{58}$ et bien d'autres grands hommes renouvelèrent le droit en joignant à la connaissance des lois celle des arts libéraux et, ouvrant ainsi la voie libre aux autres, ont gagné une gloire immortelle ? Songeons à tout le bien qu'ont apporté à l'époque moderne, à toute la gloire qu'ont gagnée Grotius, Gothofredi59, Vinnius, Binkershoek ${ }^{60}$, Schultingh ${ }^{61}$ et d'autres, qui, voyant dans les arts libéraux le moyen essentiel d'expliquer les

\section{Ibid.,f. 11.}

58. Konrad Rittershusius (1560-1613), jurisconsulte allemand, commentateur des Institutes de Justinien.

59. Il s'agit certainement du célèbre jurisconsulte Denys Godefroy (1549-1621).

60. Arnold Vinnius (1588-1657) et Corneli van Binkershoek (1673-1743), jurisconsultes hollandais.

61. Antonius Schultingh (1659-1734), qui commenta notamment les Pandectes. 
lois, ont étonné le monde par leurs succès. La plus haute antiquité peut nous fournir des exemples semblables; mais contentons-nous de prendre à témoin Cicéron, Gellius ${ }^{62}$ et Pline. Ces hommes ont tant respecté l'histoire et les antiquités qu'en évoquant certains célèbres juristes, ils plaçaient chez eux plus haut que toute chose ces connaissances dont ils avaient obtenu une grande maîtrise. Qui plus est, je sais assez l'état d'abandon et de négligence dans lequel est plongée l'histoire de la Russie, que personne, pour autant que je sache, n'a encore songé à mettre en ordre. Lorsque je retournerai dans ma patrie, si le temps, l'opportunité et mes capacités m'y autorisent, je ne manquerai certes pas d'employer tous mes efforts pour lui montrer son antiquité, sa gloire passée, sa supériorité et sa puissance, car, rejetant les opinions infondées d'aucuns, je fais mes études à la fin de servir ma patrie par tous les moyens, certain que je suis de ne pas encourir de reproches pour cela, et je peux m'en référer hardiment aux grands hommes, puisqu'ils ne blâmeraient pas mon opinion, mais confirmeront volontiers par leurs dires l'organisation de mes études. Je pourrais citer bien d'autres preuves d'une égale importance, mais le secrétariat de l'Académie des sciences verra clairement dans ce qui précède le bien-fondé de mon opinion et approuvera mes actes qui, comme je le crois, ne contiennent rien qui soit blâmable.

Quant à mon prochain départ de Strasbourg, je prie humblement le secrétariat de l'Académie des sciences de m'autoriser à passer quelque temps à Paris, tant pour la langue française que je peux oublier en pays allemand faute de pratique, que pour examiner les institutions intérieures de cette ville sous le rapport de son gouvernement. Cependant, si le secrétariat, pour des raisons qu'il connaît, ne répond pas à ma requête, du moins j'espère obtenir la permission d'aller à Göttingen et non pas à Tübingen. Tübingen convient plutôt aux théologiens qu'aux juristes; de plus, la vie dissipée des étudiants de là-bas, dont j'ai pu voir plus d'un à Strasbourg, a suscité en moi un grand mépris pour cette université. Au contraire, l'université de Göttingen, comme le reconnaissent ceux qui y ont fait longuement leurs études, présente un grand avantage sous les deux rapports, et surtout sa faculté de droit a été portée, à ce qu'on dit, à un tel degré de perfection qu'aucune autre université ne peut s'y comparer. » [Pour finir, il demande assez sèchement de l'argent pour son voyage à Paris ou à Göttingen] ${ }^{63}$

Les lettres de Polenov, on s'en doute, provoquèrent un petit scandale à l'Académie, d'autant plus que Protasov ne trouva rien de mieux que de montrer à ses collègues celle qui lui avait été adressée ${ }^{64}$, de sorte que Polenov dut ensuite battre sa coulpe et mettre son coup de sang sur le compte de sa fatigue et de sa mauvaise santé. Taubert, qui lui garda malgré tout sa faveur ${ }^{65}$, fut supplanté à l'Académie par Jacob von Stählin, nouvel homme d'influence, ce qui n'arrangea guère les affaires de

62. Aulu-Gelle.

63. PFA RAN, f. 3, inv. 1, d. 270, f. 155-155v. (rapport de Polenov daté du 7 août 1766).

64. On l'apprend d'une lettre de Polenov à Protasov du 7 janvier 1767, écrite à Göttingen : il lui reproche son geste sans guère insister, explique sa propre attitude et lui annonce qu'il a brûlé sa correspondance avant de partir pour Göttingen, RNB, f. 595, d. 90, f. 12-12v.

65. Voir la lettre de Taubert à Polenov du 3 avril 1767, RNB, f. 595, d. 93, f. 2-2v. 
Polenov. Cependant, tout ceci ne l'empêcha pas, comme la réponse de ses supérieurs tardait à venir, de partir pour Göttingen de son propre chef ${ }^{66}$.

Nous nous intéresserons moins à cet aspect anecdotique qu'à plusieurs aspects des correspondances citées qui nous paraissent très significatifs. Par-delà leur ton plein de dignité, voire de suffisance, Polenov nous expose un credo dont on trouve peu d'équivalents dans la Russie du XVIII siècle. Il fait valoir qu'il a été seul, avec l'aide des professeurs strasbourgeois, à organiser ses études. Il oppose la reconnaissance que sa valeur lui a value à Strasbourg, à l'incompréhension des Pétersbourgeois. Avec un mépris à peine dissimulé, il accuse ceux-ci d'incompétence et d'ignorance, notamment sous le rapport de l'image qu'ils se font des universités européennes. Il défend l'organisation des études à Strasbourg, en mettant un accent appuyé sur l'histoire et les antiquités (les « arts libéraux » ou les humanitas) avec une référence particulière aux humanistes français ainsi qu'aux philosophes du droit naturel du Saint Empire ; cette configuration de l'étude du droit, qui relève à la fois d'une recherche de ses origines nationales et de ses fondements philosophiques, le conduit très naturellement à placer l'université de Göttingen au-dessus de toutes les autres ${ }^{67}$. Enfin, il se présente comme patriote, et condamné à souffrir d'en être un.

Polenov veut avant tout s'affirmer comme un individu, dont l'itinéraire est original, qui, à la limite, ne le doit qu'à lui-même et qui, en tout cas, en est le seul juge ( « je sais très bien moi-même tout ce qu'il me faut»). Cette affirmation passe par deux conditions ou revendications essentielles qui sont la reconnaissance de ce qu'il est et le respect de sa liberté. La reconnaissance qu'il réclame est celle de sa compétence, de son savoir dans le domaine qu'il représente : lui seul est à même de décider de la meilleure organisation de ses études, et les seules autorités qu'il reconnaît en la matière, précisément parce qu'elles reposent sur les mêmes compétences et le même savoir, sont ses professeurs de Strasbourg, c'est-à-dire ses futurs pairs - puisqu'il se destine à la même carrière - et les grands humanistes,

66. Sur tout cet épisode, voir les lettres de Polenov à Taubert du 27 octobre 1766 (RNB, f. 595 d. 90, f. 11v.), à l'Académie du 28 octobre (PFA RAN, f. 3, inv. 1, d. 308, f. 119) et de nouveau à Taubert du 12 novembre 1766 (RNB, f. 595, d. 90,f. 12), son rapport à l'Académie du même jour (PFA RAN, f. 3, inv. 1, d. 308, f. 118-118v.), sa lettre à Protasov que nous venons de citer, ses deux lettres à Taubert écrites à Göttingen en janvier et mars 1767 (ibid.,f. 116 et 67-67v.), enfin et surtout sa lettre à Stählin du 25 janvier 1767 (RNB, f. 595, d. 90, f. 13) et à Vladimir Orlov, le même jour (ibid.,f. 12v.)

67. Dans sa lettre à Taubert du 7 août 1766, dans laquelle il demandait la permission d'aller à Paris, Polenov écrivait également : «Si vous ne jugez pas à propos de l'accorder, j'irai volontiers à Götting, à la meilleure de toutes les universités d'Allemagne pour la faculté juridique » (en français dans le texte, RNB, f. 595, d. 90, f. 11). Plus tard, lorsque Polenov se trouva enfin à Göttingen, il écrivit dans une lettre : « L'Université de Götting peut être mise au même rang des premières universités et même elle emportera peut-être sur toutes les autres dans ce qui regarde les sciences juridiques. Il y a ici dix professeurs de droit, dont chacun tient trois ou quatre collèges et les finit dans six mois ; ainsi les juristes principalement viennent de toutes les parts pour s'instruire et pour se perfectionner dans tout ce qui leur est nécessaire. » (En français dans le texte, PFA RAN, f. 3, inv. 1, d. 308, f. 67). Encore plus tard, il écrit qu'il a choisi de fréquenter seulement les collèges à Göttingen «dont les mérites sont connus partout ailleurs » (ibid., f. 116). Il appert de ses autres rapports qu'il suit (entre autres ?) un cours de droit féodal et un cours sur les Pandectes (ibid., f. 117 et 118). 
couronnés en amont par l'autorité suprême des Latins. Quant à la liberté qu'il entend défendre, il s'agit de celle de choisir : choisir l'organisation de ses études, dans la discipline qu'il a fait sienne, en usant de sa compétence, assistée par les conseils des Strasbourgeois, choisir aussi le lieu de ses études le plus convenable à ses besoins.

Dans les deux cas, Polenov retrouve certains fondements de l'institution universitaire, telle qu'elle s'est construite depuis le XIII' siècle, à savoir le libre choix des études par les étudiants et leur libre circulation entre les universités. La pérégrination académique, notamment, a toujours été, et demeure plus que jamais, au XVIII ${ }^{e}$ siècle, un élément fondamental de la vie universitaire. Dans son projet de se rendre à Paris, Polenov n'a pas besoin de chercher des exemples très loin : ses professeurs comme ses condisciples ont coutume de ce genre de voyages. Dans une lettre à Sauttersheim du $1^{\text {er }}$ janvier 1766, il lui confie : « je travaille actuellement de toutes mes forces pour qu'on me donne un certain emploi avec la permission d'aller à Paris. ${ }^{68}$ S'agissait-il de la recherche d'un emploi privé, par exemple d'instituteur ou de gouverneur, ce qui était une pratique très courante dans les milieux universitaires (aussi bien Lorenz que Koch en étaient passés par là) ? Nous l'ignorons, mais l'hypothèse est plausible.

Les valeurs dont se réclame Polenov sont donc clairement universitaires. Aussi sa défense de son plan d'études ne se limite-t-elle pas au domaine strict du droit : il en appelle aux «arts libéraux», propédeutique nécessaire à toute spécialisation, parmi lesquels l'histoire rejoint la rhétorique et les humanités classiques. Consciemment ou non, il retourne, là encore, aux sources de l'université européenne, démarche qui pourrait paraître incongrue ou naïve dans l'Europe occidentale de la fin du XVIII siècle, mais qui prend tout son poids en Russie, où l'idée même des «arts libéraux » (svobodnye nauki) n'est apparue que depuis la fin du XVII siècle, avec la création de l'Académie gréco-latino-slavonne de Moscou.

L'image que Polenov se forge de lui-même ne s'arrête pas à cette figure du membre de la corporation universitaire. Il se pose aussi en patriote et en victime vertueuse.

Nous trouvons de multiples traces de son patriotisme, probablement sincère. Par exemple, Polenov prend ombrage des préjugés dont, selon lui, les Strasbourgeois entourent la Russie. Il écrit :

«On est trop prévenu par rapport à ma patrie et sans approfondir les choses on croit tous les contes des vieilles femmes : chez nous point d'assassin, et on ne sait pas verser du sang pour contenter un faux zèle. Si ma nation est sujette à quelques défauts, qui sûrement ne sont pas d'un grand poids, les autres en ont peut-être davantage, et voilà ce que je puis dire avec la raison qu'il n'y a point d'endroit pour les étrangers où on est si bien traité et reçu que chez nous. »69

68. RNB, f. 595 , d. 90 , f. 8.

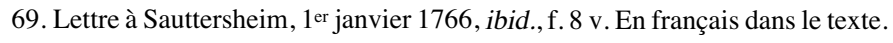


Patriote, Polenov semble l'être par conviction, ce qui ne signifie pas pour autant qu'il fasse preuve en l'occurrence d'originalité. Lorsqu'il proteste de son dévouement à la patrie («mes seules pensées inclinent à rendre des services à ma patrie », écrit-il à Protasov $)^{70}$, il reproduit évidemment un cliché : le modèle du « fils de la patrie » n'était pas seulement proposé à l'ensemble de la noblesse russe, il était aussi nourri par les petits milieux académiques et universitaires, issus de la roture, de l'armée et de la toute petite noblesse, qui s'en servaient pour s'affirmer. Lomonosov, comme on le sait, cultivait tout particulièrement cette image d'intellectuel national sorti du peuple ${ }^{71}$.

Le patriotisme de Polenov, comme chez d'autres, est orienté vers une tâche concrète : son identité d'intellectuel se cristallise dans un projet de construction nationale. Sans pousser plus avant une recherche qui reste à mener, nous nous contenterons de constater, à la lecture des lettres citées, que Polenov se sent investi d'une mission, poussé dans cette voie par Taubert qui mise visiblement gros sur son poulain. Dans une lettre à Polenov de juin 1765, Protasov lui explique ce que Taubert attend de lui : « $[\ldots]$ une fois revenu dans votre patrie, que vous puissiez non seulement enseigner votre science à d'autres avec la qualité requise, mais aussi amener tous les droits et les lois de cet État en un système bon et convenable, à l'exemple des autres États. » Et de promettre à Polenov, toujours de la part de Taubert, de lui envoyer dès l'été " tous les ukazy [sic !] et les livres s'y rapportant » ainsi que le « début déjà considérable [de ce travail] par monsieur Strube [...]. » ${ }^{72}$ De son côté, Taubert lui écrit en avril 1767 qu'il doit continuer d'étudier la Historia Juris, mais surtout, pour l'obtention du grade de docteur, « choisir une matière qui corresponde au grand dessein de notre très gracieuse Souveraine, par exemple au moyen de quelle méthode faut-il corriger les lois insuffisantes et composer de nouvelles lois, et comment les autres nations s'y sont employées dans les temps anciens et modernes. $\gg^{73}$

Par-delà, ou en-deçà de cette tâche conjoncturelle, il s'agissait de bâtir un droit national russe, ce qui conduisait Polenov, conformément à ce qu'il avait appris à Strasbourg, à rechercher les sources nationales de ce droit et à imiter en cela des juristes humanistes français du XVIe siècle (Cujas, Brisson, Hotman, etc.) dont la mention, sous sa plume, n'obéissait certes pas au hasard. De façon symptomatique, Polenov usait à propos de l'histoire russe d'expressions comme «mettre de

70. Lettre du 7 janvier 1767, à Göttingen, ibid., f. 12 .

71. Cette image n'a été aussi que trop entretenue par les historiens soviétiques après la Seconde Guerre mondiale avec les distorsions nationalistes qu'on connaît. Cela ne signifie pas pour autant qu'elle soit fausse. L'ouvrage de Mihail M. Strange, en particulier, Demokratičeskaja intelligencija Rossii v XVIII veke, op. cit., a ouvert des pistes importantes dans cette direction, malgré les orientations idéologiques qui l'obèrent lourdement.

72. PFA RAN, f. 3, inv. 1, d. 270, juin 1765, f. 97-97v. De son côté, Polenov fut évidemment sensible aux perspectives que Taubert faisait miroiter à ses yeux : « On me fait des reproches et des menaces d'un côté et d'autre on m'assure de la protection en promettant d'avoir tous les soins possibles pour moi. Je m'y fie et compte pour sur qu'on me tiendra la parole [...]. », écritil en français à Sauttersheim le 8 octobre 1766 (RNB, f. 575,d.90,f. 11v.)

73. Ibid., d. 93, f. 2-2v., lettre datée du 3 avril 1767 (a.s.). 
l'ordre » (privedenie V porjadok) ou «abandon et négligence » (zapuščenie $i$ nebreženie) qui s'appliquaient parfaitement - et qui avaient été appliquées - au droit de l'Empire de Russie : c'est que, dans son esprit, il s'agissait d'une seule et même démarche, celle de la découverte et de la mise en forme des lois passées et existantes. Chargé par son protecteur, avant d'être ainsi sollicité par l'actualité, de rédiger un travail historique et comparatif sur le droit, travail dont on ne sait pas grand-chose, mais qu'on peut présumer propédeutique à sa mission future ${ }^{74}$, Polenov semblait y mettre beaucoup d'importance et de soin. «C'est une affaire », écrivait-il à Protasov, « qui tant par sa difficulté que par son importance surpasse presque mes forces, c'est une affaire, dis-je, qui me fait trembler d'avance. Né pour servir ma patrie, j'employerai tout ce qui dépendra de moi, pour lui rendre les services dus [...]. » ${ }^{75}$. Et un mois après, il confiait à Sauttersheim : « Je travaille actuellement de toutes mes forces à un petit ouvrage qu'on exige de moi pour preuve de mes succès dans les études et je l'arrange à mon gout [...]. »76 Dans une lettre à Taubert, dans laquelle il lui rendait compte de cette tâche, il livrait quelques détails intéressants sur ce travail :

« Il suffira, je crois, de choisir quelques matières les plus intéressantes du droit civil, criminel et ecclésiastique, dont je traiterai selon l'ordre reçu. La loi de Rome gouverne presque toute l'Europe, c'est pourquoi il est bien juste si je la prends pour mon sujet : outre cela je n'oublierai non plus de faire mention de quelques cas particuliers de la jurisprudence Françoise et Allemande, qui sont tout à fait contraires au droit Romain et dont l'autorité malgré tous les efforts n'a pu s'en garantir. Quant à notre jurisprudence, elle a beaucoup de ressemblance à celle de Rome et ce n'est pas étonnant, on n'a qu'à lire la préface du Code Alexis, on en trouvera bientôt la cause. $\gg^{77}$

Cette comparaison entre l'Uloženie de 1649 et le droit romain reste mystérieuse et pour le moins surprenante. Probablement, Polenov traçait un parallèle entre le rôle de Justinien et celui d'Alexis dans l'instauration d'un code universel, dont l'action devait s'étendre à tous les sujets du monarque, comme le préambule du code d'Alexis le précisait explicitement ; d'autre part, Polenov pouvait avoir en vue une similitude des méthodes, consistant, dans le cas russe, à mettre en harmonie les anciennes législations, le droit canon, avec les lois édictées depuis le règne de Michel Romanov sans que les premières fussent sacrifiées. Cette hypothèse est d'autant plus probable que Strube de Piermont n'avait pas affirmé autre chose dans ses recherches sur les anciennes lois russes : après avoir cité plusieurs États, notamment dans le Saint Empire, qui avaient su adapter leurs anciennes lois sans les

74. Ce document, dont on sait que Polenov en envoya au moins le début à Taubert (voir sa lettre à Taubert du 11 juin 1766: «J'ai l'honneur de vous envoyer le commencement de mon ouvrage », ibid., f. 10), présenterait certainement beaucoup d'intérêt, mais il demeure pour le moment introuvable.

75. Lettre du 29 mars 1766, RNB, f. 595, d. 90, f. 9. En français dans le texte.

76. Ibid., f. 9v., lettre du 24 avril 1766. En français dans le texte.

77. Ibid., f. 8v.-9, lettre du 24 mars 1766. En français dans le texte. 
abolir, il avait écrit : «Il est clair par ce Préambule qui se trouve à la tête de l'Oulojénié que le Tsar Alexis Mikhailovicz avoit ordonné exprès de restreindre les loix contenues dans ce code aux cas qui n'avaient pas été décidés par les loix antérieures. $\gg^{78}$ Pour résumer, nous dirions que le projet de Polenov, pour autant que nous pouvons en juger, relevait à la fois de la recherche historique et de l'étude comparative, puisqu'il s'agissait de revivifier les sources du droit russe et de codifier la législation en vigueur à la lecture du droit romain. Ce souci d'acquérir sciemment un savoir puisé à l'extérieur de la Russie en remontant aux sources antiques, et de le transférer en Russie en recherchant sa propre antiquité nous paraît très caractéristique de la façon dont les intellectuels russes du XVIII ${ }^{e}$ siècle s'efforcèrent de construire une culture nationale, dans des domaines comme la littérature, la langue littéraire et savante, l'historiographie ou les arts. ${ }^{79}$ Polenov ne semblait pas faire autre chose dans sa spécialité.

Intellectuel et patriote, Polenov se présentait aussi comme une victime de sa propre vertu. Ce rôle du solitaire abandonné de tous s'éclaire mieux si nous nous penchons sur les relations qui l'unissaient à son ami Sauttersheim. Jean-Ignace Sauttermeister de Sauttersheim (1738-1767) était un faux baron hongrois, qui avait fui Vienne à cause de ses dettes et qui avait réussi à se lier avec Jean-Jacques Rousseau en le croisant à Môtiers, après quoi il entretint avec lui une correspondance entre avril 1763 et $1766^{80}$. Il vouait un culte au grand homme et, ce qui est le plus intéressant, présentait ce culte comme s'il était répandu à Strasbourg. Par exemple, dans sa lettre à Jean-Jacques du 16 janvier 1765, il évoquait des amis de Strasbourg qui «ont puisé la vertu dans vos ouvrages et leurs actions ne démentent pas vos doctrines. ${ }^{81}$ Le 20 février 1765, il lui écrivit qu'il se trouvait dans la bibliothèque d'un Strasbourgeois « où toutes vos principes sont regardées comme des loix, où on n'aime parler que de Vous et de vos écrites, où on ne souffre enfin, que ceux qui sont vertueux ou qui veulent l'être. ${ }^{82}$

Malheureusement, Polenov ne conserva pas les lettres de son ami. Mais les copies des siennes à Sauttersheim nous donnent à comprendre qu'ils partageaient entre eux ce culte de Rousseau. Au moment où celui-ci vint à Strasbourg, Sauttersheim était déjà à Paris, et Polenov en rendit fidèlement compte au pseudo-

78. «Loi Russienne donnée aux habitants de Novgorod par le grand duc Jaroslaf et ses fils en $1017 »$, op. cit., f. 8 , note i.

79. Ce n'est pas le lieu ici de nous étendre sur ce sujet qui n'est certes pas inconnu dans l'historiographie russe et occidentale, mais qui nécessiterait des études plus approfondies que celles qui existent. La seule synthèse qui ait été tentée à ce jour dans ce domaine est celle de Hans Rogger, National consciousness in eighteenth-century Russia, Cambridge, Mass., Harvard University Press, 1960.

80. Dans la Correspondance complète de Jean-Jacques Rousseau (éd. par Theodore Besterman, Voltaire Foundation, Oxford), les lettres adressées par Sauttersheim sont publiées dans les volumes XVI à XXX.

81. Lettre 3887, vol. XXIII.

82. Lettre 4046, ibid. 
baron, évoquant avec une ironie entendue les rumeurs dont l'«illustre ami »du Hongrois faisait l'objet dans la société strasbourgeoise :

«Je serois fort charmé de vous revoir, il me paroit que Mr Rousseau, votre illustre ami, en sera la cause. Dès son arrivée à Strasbourg, tout le monde étoit curieux de le voir, on en jugeait selon ce qu'on étoit soi-même. On disoit mille bêtises et mille mensonges, chez l'un il passoit pour un philosophe, chez l'autre pour un fou, chez le troisième pour un misantrope, la populace le regarde comme un sorcier, principalement par rapport à son habit car l'extérieur suffit pour ces gens pour qu'ils s'en fassent ou un idole ou un monstre. »

Et voici ce que Polenov écrit à propos du Devin du village qui fut représenté à Strasbourg en présence de son auteur :

« J'ai bien reconnu l'auteur à la pièce. Il y avait des sentimens dignes d'un homme vertueux et aussi de l'humanité : mais hélas! on s'en moque de notre tems. À cette heure, la fureur se passa et on n'en parle plus, la curiosité s'évanouit et le laisse tranquille. Je l'ai vu quelquefois et si vous viendrez ici, j'espère obtenir une grâce de votre amitié, que vous me trouverez l'occasion d'avoir l'honneur de lui parler. ${ }^{83}$

Par la suite, à cause d'une affaire de dette quelque peu obscure, Polenov faillit se quereller avec Sauttersheim et lui écrivit des lettres grandiloquentes, dans lesquelles on trouve des expressions comme celles-ci : «je ne suis ni usurier, ni marchand, mais votre ami, par conséquent je n'ai point de compte à rendre. Votre seule parole étoit plus forte que toutes les assurances d'un autre; aussi je vous ai regardé toujours comme mon ami. » «La question a si peu d'importance pour un honnête homme qu'il ne vaut pas la peine d'en parler. » « Pardonnez à ma franchise et à ma sincérité ces expressions rudes. » «Vous verrez dans la suite qu'un homme comme moi mérite plus d'attention que vous ne faites pas. » « Le climat du Nord, tout rude qu'il est, en me formant ne m'a pas ôté toute la sensibilité, un cœur simple et naturel à la vue d'un soupçon si énorme et si outrageant ne peut pas être insensible. » Et d'évoquer son «amour de la vérité et de l'humanité », et de se proclamer avant tout « honnête homme ${ }^{84}$. Encore plus tard, il écrivait en français à Stählin, qui, nouvel homme fort à l'Académie, avait contribué à ses ennuis du moment ${ }^{85}$ et avec lequel il tentait de se raccommoder :

« Depuis mon départ, il n’y avait, pour vous dire le vrai, que des disgrâces et des malheurs pour moi, dont je ne vois pas encore la fin [...]. Combien de fois n'ai-

83. RNB, f. 595 , d. 90 , f. 7v. (lettre du 25 novembre 1765). En français dans le texte.

84. Lettres en français du 23 mai et du 17 juin 1766, ibid., f. 9v.-10.

85. On le sait par la correspondance de Polenov qui laisse deviner les informations qu'il recevait, notamment de Protasov. À ce dernier, il écrit en effet le 7 janvier 1767: «Je vous prie humblement de représenter à monsieur Stählin que s'il a trouvé dans mon rapport certaines expressions, je ne manquerai pas de m'excuser auprès de lui comme il le convient [...]. » (ibid., f. 12). 
je pas détesté cette malheureuse passion qui m'encouragea de quitter ma patrie et pour quoi faire? Pour ruiner ma santé, pour essuyer mille dangers sur la mer et enfin pour être persécuté comme un criminel, ce sont les beaux fruits de mon voyage et de mes études ; peut-on croire que quelques paroles précipitées puissent attirer tant de disgrâces à un honnête homme : tout le monde est contre moi, qui suis sans amis, sans protecteurs. » ${ }^{86}$

Et enfin à Taubert, pour lui redemander protection, quitte à l'assurer dans une autre lettre de sa fidélité, même si l'étoile de celui-ci avait pâli depuis que le secrétariat de l'Académie avait été supprimé et que Vladimir Orlov en avait pris la direction : « Je me flatte que je ne serai jamais abandonné de mon bienfaiteur; en perdant cette esperance je perdrai tout et il ne restera pour moi que le seul desespoir d'etre en prö̈e à la persécution. $»^{87}$

Nous croyons voir dans ces attitudes non pas, certes, une imitation pure et simple du citoyen de Genève, mais de possibles accents rousseauistes. La vertu, la franchise et la simplicité, la marginalité, l'image de la persécution, tels étaient, sans doute, les traits du personnage que Polenov se composait non sans affectation, traits qu'il avait peut-être perçus chez Rousseau. Ils lui donnaient une contenance supplémentaire, d'autant plus qu'ils se conjuguaient avec ses origines russes, non moins « simples et naturelles » dans l'imaginaire européen, et avec sa fierté nationale. Qui plus était, la position de la marginalité réelle ou imaginaire était suffisamment répandue dans l'Europe de la fin du XVIII e siècle pour qu'un petit noble russe, plus ou moins déclassé et incertain de son avenir, s'y reconnût et y trouvât à se conforter. Les habits de Rousseau, dont il donnait du reste une description dans sa lettre à Sauttersheim, se combinaient avec ceux de l'universitaire en herbe pour former, somme toute, une image assez cohérente et adaptée aux petits ou grands drames qu'il avait à affronter. Il ne se privait pas pour s'en servir, notamment, dans les situations de conflit. À Protasov, dans une lettre déjà citée, dans laquelle il tentait d'effacer la mauvaise impression laissée par ses missives provocatrices, il écrivait par exemple : «Au reste, monsieur, ma sincérité ne doit pas vous être importune. Je suis né avec elle, de sorte que l'on ne doit pas me l'incriminer. ${ }^{88} \mathrm{Sa}$ « façon de penser simple et naturelle » ne pouvait, selon lui, offenser quiconque, parce que, précisément, elle relevait de la nature, de sorte que la simplicité (la nature), dont on voit bien qu'elle se confondait chez lui avec l'idée de droit naturel, épaulait la science lorsqu'il en était besoin. C'est un peu cette double identité que Polenov déclinait à Protasov, dans cette définition égotiste, si peu conforme aux usages de ses confrères et de son époque en Russie :

« Je connais ma charge, j'aime ma patrie, j'honore l'honnêteté chez les autres et l'observe moi-même ; qui plus est, j'ai une certaine notion de la supériorité des

86. Lettre en français du 25 janvier 1767, ibid., f. 12v.-13

87. Lettre écrite en français à Göttingen en 1767 ; PFA RAN, f. 2, inv. 1, d. 308, f. 67-67v.

88. Lettre à Protasov du 7 janvier 1767, Göttingen, RNB., f. 595, d. 90, f. 12. Souligné par nous. 
sciences, ce qui éveille en moi mon inclination pour elles; en un mot, la vie honnête et les sciences sont pour moi deux trésors et je n'épargne pas mes efforts pour m'affermir dans la première et acquérir les secondes avec le temps. ${ }^{89}$

Clerc, patriote, vertueux : cette image narcissique ne pouvait manquer de se heurter aux hiérarchies et aux mœurs de l'Empire russe, fussent-elles celles de l'Académie des sciences ${ }^{90}$. Mais, par-delà le cas particulier de Polenov, c'est, au fond, sa condition même d'intellectuel que ce conflit mettait au jour, sous une forme certainement plus brutale, plus raccourcie, mais aussi plus nette qu'ailleurs en Europe : car le sentiment du bon droit, si flagrant chez Polenov, lui venait de sa certitude de vivre selon des valeurs qui, précisément, transcendaient sa condition, car elles étaient universelles, mieux, le faisaient accéder à la vérité universelle. C'est à ce titre que la modeste histoire de Polenov pourrait nous aider à mieux comprendre la formation des intellectuels en Europe.

Centre d'études du monde russe, soviétique et post-soviétique

54, boulevard Raspail

75006 Paris

Université de Genève

Faculté des lettres

3 , rue de Candolle

CH-1205 Genève

berelowi@ehess.fr

90. Faute de place, nous n'évoquons guère le séjour de Polenov à Göttingen en 1767, l'issue de son conflit avec l'Académie, notamment avec Stählin, ni son sort ultérieur après son retour de Göttingen la même année, dont on trouvera des récits dans les études citées ci-dessus. Qu'il suffise de dire que sa carrière fut honorable, mais sans doute pas à la hauteur de ses ambitions, car son œuvre de juriste passa inaperçue, malgré les qualités en tous points remarquables de sa réponse au concours de la Société d'économie. 